IUHET 395

August 1998

\title{
Hydrogen and Antihydrogen Spectroscopy for Studies of CPT and Lorentz Symmetry ${ }^{1}$
}

\author{
Robert Bluhm ${ }^{a}$, V. Alan Kosteleckýb ${ }^{b}$ and Neil Russell ${ }^{b}$ \\ ${ }^{a}$ Physics Department, Colby College, Waterville, ME, 04901 U.S.A. \\ ${ }^{b}$ Physics Department, Indiana University, Bloomington, IN, 47405 U.S.A.
}

\begin{abstract}
A theoretical study of possible signals for CPT and Lorentz violation arising in hydrogen and antihydrogen spectroscopy is described. The analysis uses a CPT- and Lorentz-violating extension of quantum electrodynamics, obtained from a general Lorentz-violating extension of the minimal standard model with both CPT-even and CPT-odd terms. Certain 1S-2S transitions and hyperfine Zeeman lines exhibit effects at leading order in small CPT-violating couplings.
\end{abstract}

\section{INTRODUCTION}

At presently accessible energy scales, which are determined by the electroweak scale $m_{W}$ and are small relative to the Planck mass $M_{P}$, the predictions of the minimal $\mathrm{SU}(3) \times \mathrm{SU}(2) \times \mathrm{U}(1)$ standard model appear to be in agreement with nature. However, at scales closer to $M_{P}$ this model is expected to be superseded by a fundamental theory that also combines quantum mechanics and gravitation in a consistent way. The fundamental theory is likely to involve qualitatively new physics as, for example, occurs in string $(\mathrm{M})$ theory at the Planck scale. Associated low-energy signals may exist. However, approximately 17 orders of magnitude separate $m_{W}$ from $M_{P}$, so effects specific to the fundamental theory and accessible via existing techniques are likely to be heavily suppressed. Experiments that search for effects forbidden in the usual renormalizable gauge theories and that are of high precision are therefore of particular interest.

In this talk, the idea is considered that the new physics includes a spontaneous violation of Lorentz symmetry [1]. If a theory with Lorentz-covariant dynamics involves Lorentz-tensor interactions acting to destabilize the naive vacuum, some finite Lorentz-tensor expectation values may arise. This can occur in some string theories, for instance. In the low-energy theory at the level of the standard model, apparent Lorentz violations would ensue if the orientation of the tensor expectation values includes the physical four spacetime dimensions.

The CPT theorem connects Lorentz transformations to the discrete charge-conjugation $(\mathrm{C})$, parity-reflection $(\mathrm{P})$, and time-reversal (T) transformations [2]. It implies that all local relativistic quantum field theories satisfying mild technical assumptions are invariant under CPT. This suggests that both CPT and Lorentz violations represent unconventional and potentially observable effects emerging from a fundamental theory. However, the heavy suppression expected from the hierarchy between $m_{W}$ and $M_{P}$ implies that detection of these effects would be feasible only in particularly sensitive experiments.

\section{EXTENDED QUANTUM ELECTRODYNAMICS}

At the level of the minimal standard model, the consequences of spontaneous Lorentz and CPT breaking can be investigated by incorporating possible terms that would represent violations of these symmetries. There exists a general Lorentz-violating extension of the minimal $\mathrm{SU}(3) \times \mathrm{SU}(2) \times \mathrm{U}(1)$ standard model [3]. It includes both CPT-even and CPT-odd terms. To date, it appears to be the sole existing candidate for a consistent standard-model extension based on a microscopic description of CPT and Lorentz violation. In any event, this theory is necessarily

1) Presented by V.A.K. at the 1998 Conference on Trapped Charged Particles and Fundamental Physics, Pacific Grove, California, August-September 1998 
the low-energy limit of any fundamental theory that contains the standard model and incorporates spontaneous CPT and Lorentz violation.

The standard-model extension is theoretically attractive for several reasons. For one, the usual structure of the gauge invariances and the spontaneous gauge-symmetry breaking are unaffected, and energy and momentum are conserved provided the Lorentz symmetry breaking produces position-independent expectation values. Also, standard quantization methods apply, and the extension is hermitian and power-counting renormalizable. Even though Lorentz symmetry is spontaneously broken, various desirable features of Lorentz-covariant theories such as positivity of the energy and microcausality are expected to persist [3]. This is largely a result of the Lorentz covariance of the underlying fundamental theory and the conventional quantum description. In fact, invariance under rotations or boosts of the observer's inertial frame (observer Lorentz transformations) is retained even at the level of the standard-model extension. Only rotations or boosts of particles and localized field distributions (particle Lorentz transformations) introduce Lorentz breaking, as a result of couplings to the tensor vacuum expectation values.

Details of the construction and the specific form of the standard-model extension, including both CPT-even and CPT-odd terms, are provided in the literature [3]. Various limits of this theory are of direct relevance to experiments testing aspects of quantum electrodynamics (QED). In this talk, attention is primarily given to the special limit that produces a CPT- and Lorentz-violating theory for a charged fermion interacting via the electromagnetic force [3]. As an explicit example, here are the terms appearing in the lagrangian extension of the usual quantum theory of photons, electrons, and positrons. In units with $\hbar=c=1$, the standard QED lagrangian is

$$
\mathcal{L}^{\mathrm{QED}}=\bar{\psi} \gamma^{\mu}\left(\frac{1}{2} i \stackrel{\leftrightarrow}{\partial_{\mu}}-q A_{\mu}\right) \psi-m \bar{\psi} \psi-\frac{1}{4} F_{\mu \nu} F^{\mu \nu}
$$

In the fermion sector, there are two CPT-breaking terms:

$$
\mathcal{L}_{e}^{\mathrm{CPT}}=-a_{\mu} \bar{\psi} \gamma^{\mu} \psi-b_{\mu} \bar{\psi} \gamma_{5} \gamma^{\mu} \psi \quad, \quad,
$$

while there is one possibility in the photon sector:

$$
\mathcal{L}_{\gamma}^{\mathrm{CPT}}=\frac{1}{2}\left(k_{A F}\right)^{\kappa} \epsilon_{\kappa \lambda \mu \nu} A^{\lambda} F^{\mu \nu} .
$$

The possible Lorentz-violating but CPT-preserving terms in the fermion sector are:

$$
\mathcal{L}_{e}^{\text {Lorentz }}=c_{\mu \nu} \bar{\psi} \gamma^{\mu}\left(\frac{1}{2} i \stackrel{\leftrightarrow}{\partial^{\nu}}-q A^{\nu}\right) \psi+d_{\mu \nu} \bar{\psi} \gamma_{5} \gamma^{\mu}\left(\frac{1}{2} i \overleftrightarrow{\partial^{\nu}}-q A^{\nu}\right) \psi-\frac{1}{2} H_{\mu \nu} \bar{\psi} \sigma^{\mu \nu} \psi
$$

while the only possibility in the photon sector is:

$$
\mathcal{L}_{\gamma}^{\text {Lorentz }}=-\frac{1}{4}\left(k_{F}\right)_{\kappa \lambda \mu \nu} F^{\kappa \lambda} F^{\mu \nu} .
$$

In the above expressions, the unconventional coupling coefficients govern the magnitude of the CPT- and Lorentzviolating effects and are expected to depend on the small ratio $m_{W} / M_{P}$. Note that all the extra couplings are hermitian. It can be shown using field redefinitions that some coupling-coefficient components are physically unobservable. The reader is referred to the literature [3] for details about this and other issues, and for more information about the notation used above.

\section{OVERVIEW OF SOME EXPERIMENTAL TESTS}

Most experiments testing Lorentz invariance or CPT symmetry are likely to be insensitive to the extra couplings in the standard-model extension due to the expected heavy suppression factors. A few experiments of exceptional sensitivity could bound or in principle detect these effects despite the suppression. In such cases, the standardmodel extension can be used as a quantitative theoretical guide to potential experimental signals. It also offers the possibility of analyzing and comparing bounds on CPT and Lorentz violation arising from different experiments.

At present, implications of the standard-model extension have been studied for CPT and Lorentz tests that involve: observations of neutral-meson oscillations [4-7], measurements of particle and antiparticle properties in Penning traps $[8,9]$, spectroscopic comparisons of hydrogen and antihydrogen [10,11], determination of photon properties [3], and baryon-number generation [12]. A variety of additional studies are in progress, notably one [13] establishing the implications for the standard-model extension of high-precision clock-comparison experiments [14].

This section of the talk provides a short summary of a subset of the results obtained. The use of hydrogen and antihydrogen spectroscopy to test CPT and Lorentz symmetries is described in the following three sections.

The flavor oscillations of certain neutral-meson systems provide a valuable interferometric tool for studying CP violation. The effective hamiltonian for the time evolution of a $P$-meson state, where $P$ represents one of the neutral 
$K, D, B_{d}$, or $B_{s}$ mesons, depends on two kinds of (indirect) $\mathrm{CP}$ violation. The first involves $\mathrm{T}$ violation with $\mathrm{CPT}$ invariance and is conventionally described with a complex parameter $\epsilon_{P}$. The second involves CPT violation with $\mathrm{T}$ invariance and is described with a complex parameter $\delta_{P}$. The standard-model extension can be used to derive an expression for $\delta_{P}[7]$.

It turns out that flavor oscillations in neutral- $P$ systems are sensitive to only one type of CPT-violating term in the standard-model extension, $-a_{\mu}^{q} \bar{q} \gamma^{\mu} q$, where $q$ is a quark field and $a_{\mu}^{q}$ is a spacetime-constant coupling coefficent with value dependent on the quark flavor $q$. None of the other experiments discussed in this talk involve flavor changes, and it has been shown that as a result these other experiments are insensitive to $a_{\mu}^{q}$-type coupling coefficients. In this respect, the bounds on CPT violation from neutral-meson tests of CPT are entirely disjoint from those of other experiments.

In the observer frame in which the Lorentz-violating coupling coefficients are defined, denote the $P$-meson fourvelocity by $\beta^{\mu} \equiv \gamma(1, \vec{\beta})$. Then, at leading order in all the standard-model coupling coefficients, the expression for $\delta_{P}$ is $[7]$

$$
\delta_{P} \approx \frac{\gamma\left(\Delta a_{0}-\vec{\beta} \cdot \Delta \vec{a}\right)}{\Delta m} i \sin \hat{\phi} e^{i \hat{\phi}}
$$

In this equation, $\Delta a_{\mu} \equiv a_{\mu}^{q_{2}}-a_{\mu}^{q_{1}}$, where $q_{1}$ and $q_{2}$ represent the valence-quark flavors in the $P$ meson. Also, $\hat{\phi} \equiv \tan ^{-1}(2 \Delta m / \Delta \gamma)$, where the mass and decay-rate differences between the $P$-meson eigenstates are, respectively, $\Delta m$ and $\Delta \gamma$.

The expression for $\delta_{P}$ implies a proportionality between the real and imaginary parts of $\delta_{P}[5]$. Note that the magnitude of $\delta_{P}$ can vary with $P$ because the couplings $a_{\mu}^{q}$ are flavor dependent [5], so the magnitude of CPTviolating effects may differ in distinct neutral-meson systems. For instance, the magnitude of CPT violation might grow with the mass of the quarks involved, as the Yukawa couplings do in the standard model. Also, the explicit dependence in Eq. (6) of $\delta_{P}$ on the boost magnitude and orientation implies several types of potentially observable effect including, for instance, larger CPT-violating effects in boosted mesons [7]. Experiments involving mesons with different momenta may therefore have different CPT reaches. The best reported bounds to date come from the kaon system [4]. Recently, two CERN experiments [6] have obtained results for the $B_{d}$ system, following the observation [5] that existing data already suffice to yield CPT limits. Other studies are ongoing.

A number of experiments that test CPT and Lorentz symmetries in a different way have been performed with the goal of comparing particle and antiparticle properties. An important technique is the use of a Penning trap to confine single particles over relatively large time scales while high-precision measurements are taken of properties such as anomaly and cyclotron frequencies [8]. Experiments of this type can constrain, for example, the coupling coefficients in the fermion sector of the extended QED. Possible observable signals in the context of this theory, the corresponding relevant figures of merit, and the associated CPT and Lorentz reaches have been obtained [9]. As just one example, using existing technology and implementing a relatively minor change in experimental procedure, Penning-trap experiments comparing the anomalous magnetic moments of electrons and positrons could place a bound of roughly $10^{-20}$ on a figure of merit involving the spatial components of the coefficient $b_{\mu}$.

The extra terms (3) and (5) in the QED extension represent modifications to photon properties. It turns out that the ensuing generalized Maxwell equations describe two independent propagating degrees of freedom as in the conventional case [3]. Typically, however, each has a distinct dispersion relation, which implies several interesting effects. For example, the vacuum becomes birefringent, so that in the presence of the CPT and Lorentz violation an electromagnetic wave propagating in the vacuum exhibits properties similar to those displayed by conventional radiation traveling in an optically anisotropic and gyrotropic transparent crystal having spatial dispersion of the axes. Behavior of this type can be constrained from the observed absence of birefringence on radio waves propagating over cosmological distances. The components of the CPT-odd coefficient $\left(k_{A F}\right)_{\mu}$ are presently bounded to $\lesssim 10^{-42} \mathrm{GeV}$ $[15,16]$, although a disputed claim [17,18] exists for a nonzero effect with $\left|\vec{k}_{A F}\right| \sim 10^{-41} \mathrm{GeV}$. The rotation-invariant irreducible component of the CPT-even coefficient $\left(k_{F}\right)_{\kappa \lambda \mu \nu}$ is bounded to $\lesssim 10^{-23}$ by cosmic-ray existence [19] and other experiments. The rotation-violating irreducible components of $\left(k_{F}\right)_{\kappa \lambda \mu \nu}$ could in principle be bounded to about $10^{-27}$ with existing techniques seeking cosmological birefringence [3], but no actual limit has been obtained to date.

The CPT-even term in Eq. (5) introduces no theoretical difficulties. However, the CPT-odd term in Eq. (3) can generate negative contributions to the energy [15]. This may represent a theoretical difficulty and indicates $\left(k_{A F}\right)^{\kappa}$ vanishes [3], which would be in agreement with the tight experimental bound from cosmological birefringence. It can be argued that a zero value of $\left(k_{A F}\right)_{\mu}$ is acceptable theoretically despite the possibility of radiative corrections from diagrams involving the CPT-violating couplings in the fermion sector because the one-loop effects are finite. 


\section{S-2S SPECTROSCOPY IN FREE HYDROGEN AND ANTIHYDROGEN}

The remainder of this talk addresses the possibility of searching for CPT and Lorentz violations by making highprecision comparisons of the spectra of hydrogen and antihydrogen [11]. The feasibility of the idea of comparative tests [10] has received a boost following the recent production and observation of antihydrogen [20,21], and several proposals for antihydrogen spectroscopy have been advanced. In the near future, the antihydrogen fine structure and Lamb shift may be obtained within a few percent by observations on a relativistic antihydrogen beam [22]. A more ambitious goal is to measure the two-photon $1 \mathrm{~S}-2 \mathrm{~S}$ transition in antihydrogen, which is expected to have a natural linewidth of only $1.3 \mathrm{~Hz}$ and is therefore a promising candidate for high-precision spectroscopy. Proposed experiments [23] would provide a comparison of the 1S-2S transitions in spin-polarized hydrogen and antihydrogen confined within a magnetic trap. For hydrogen, a cold atomic beam has been used to measure the 1S-2S transition frequency to 3.4 parts in $10^{14}$ [24], while trapping techniques have yielded a frequency precision of about $10^{-12}$ [25]. A limiting accuracy of about $10^{-18}$ may be attainable [26].

A theoretical analysis of signals for CPT and Lorentz violations in hydrogen and antihydrogen spectroscopy is feasible [11] in the context of the QED extension described in the second part of this talk. In this section, possible effects on the free-atom 1S-2S transition are considered. These are relevant, for example, to the experiments with cold atomic beams of hydrogen [24]. The next section treats the trapped-atom case. A detailed theoretical treatment of the proposed experiments with relativistic beams [22], which are expected to have significantly poorer frequency resolutions than those based on other techniques, remains to be performed and is not discussed here. Note, however, that all the experimental situations discussed below are sensitive only to spatial or mixed spatio-temporal components of the CPT- and Lorentz-violating couplings in the comoving Earth frame, whereas a boost can induce sensitivity to purely timelike components and can enhance CPT- and Lorentz-violating effects [7].

To calculate effects on the free-atom $1 \mathrm{~S}$ and $2 \mathrm{~S}$ energy levels, the modified Dirac equation for a four-component electron field $\psi$ in the proton Coulomb potential

$$
A^{\mu}=\frac{|e|}{4 \pi r}(1, \overrightarrow{0})
$$

is needed. The desired equation is found from Eqs. (1), (2), and (4) to be

$$
\left(i \gamma^{\mu} D_{\mu}-m_{e}-a_{\mu}^{e} \gamma^{\mu}-b_{\mu}^{e} \gamma_{5} \gamma^{\mu}-\frac{1}{2} H_{\mu \nu}^{e} \sigma^{\mu \nu}+i c_{\mu \nu}^{e} \gamma^{\mu} D^{\nu}+i d_{\mu \nu}^{e} \gamma_{5} \gamma^{\mu} D^{\nu}\right) \psi=0
$$

where $m_{e}$ is the electron mass and the covariant derivative is

$$
i D_{\mu} \equiv i \partial_{\mu}-q A_{\mu}
$$

with the electron charge being $q=-|e|$. Both a free electron and a free proton have distinct CPT- and Lorentzviolating coupling coefficients in the typical case [3,9], so superscripts $e$ have been added to the couplings in Eq. (8). In what follows, the corresponding couplings for a free proton are denoted by $a_{\mu}^{p}, b_{\mu}^{p}, H_{\mu \nu}^{p}, c_{\mu \nu}^{p}, d_{\mu \nu}^{p}$. Note that, as mentioned following Eq. (5), certain combinations of the electron and proton couplings can be shown on general grounds to be physically unobservable [3]. This is true, for example, of the coefficients $a_{\mu}^{e}$ and $a_{\mu}^{p}$. Although all couplings are kept explicitly in the derivations that follow, it is to be expected that the ensuing possible spectroscopic signals in hydrogen and antihydrogen are independent of the unobservable couplings.

Since the coupling coefficients are expected to be highly suppressed, it is reasonable to calculate the dominant effects on the hydrogen and antihydrogen spectra via perturbation theory in relativistic quantum mechanics. The relevant unperturbed hamiltonians and the corresponding eigenstates are identical for hydrogen and antihydrogen, as are all perturbative effects from conventional quantum electrodynamics. The unconventional coupling coefficients introduce hermitian perturbations that can differ for hydrogen and antihydrogen. For the electron and positron, the explicit forms of these perturbations follow from Eq. (8) after application of suitable field redefinitions to obtain the hamiltonian and, for the positron, a standard charge-conjugation procedure [9]. The CPT and Lorentz violations from the proton sector also produce energy perturbations, which at leading order can be derived using relativistic two-fermion techniques [27].

In what follows, the uncoupled angular-momentum quantum numbers for the S-state electron/positron and for the proton/antiproton are denoted by $J=1 / 2$ and $I=1 / 2$, respectively. Their components along the spin-quantization axis are $m_{J}, m_{I}$, and the corresponding basis states are denoted $\left|m_{J}, m_{I}\right\rangle$. Note that distinct real experiments are likely to involve different spin-quantization axes relative to any single specified inertial frame, so comparisons between various experiments may require care in allowing for possible geometrical factors.

The result of the perturbative calculation is that the $1 \mathrm{~S}$ and $2 \mathrm{~S}$ levels in hydrogen are shifted by identical amounts $\Delta E^{H}[11]:$ 


$$
\begin{aligned}
\Delta E^{H}\left(m_{J}, m_{I}\right) \approx\left(a_{0}^{e}+a_{0}^{p}-c_{00}^{e} m_{e}-c_{00}^{p} m_{p}\right) & +\left(-b_{3}^{e}+d_{30}^{e} m_{e}+H_{12}^{e}\right) \frac{m_{J}}{\left|m_{J}\right|} \\
& +\left(-b_{3}^{p}+d_{30}^{p} m_{p}+H_{12}^{p}\right) \frac{m_{I}}{\left|m_{I}\right|}
\end{aligned}
$$

where $m_{p}$ is the proton mass.

A similar calculation for antihydrogen also yields equal $1 \mathrm{~S}$ and $2 \mathrm{~S}$ level shifts $\Delta E^{\bar{H}}$, given by Eq. (10) with the substitutions

$$
a_{\mu}^{e} \rightarrow-a_{\mu}^{e} \quad, \quad d_{\mu \nu}^{e} \rightarrow-d_{\mu \nu}^{e} \quad, \quad H_{\mu \nu}^{e} \rightarrow-H_{\mu \nu}^{e} \quad ; \quad a_{\mu}^{p} \rightarrow-a_{\mu}^{p} \quad, \quad d_{\mu \nu}^{p} \rightarrow-d_{\mu \nu}^{p} \quad, \quad H_{\mu \nu}^{p} \rightarrow-H_{\mu \nu}^{p} .
$$

Note that in all these expressions the leading-order contributions from the proton/antiproton have the same mathematical form as those from the electron/positron.

The electron (positron) and proton (antiproton) angular momenta are coupled through the hyperfine interaction. The relevant basis states are thus linear combinations $\left|F, m_{F}\right\rangle$ of the $\left|m_{J}, m_{I}\right\rangle$ states, where $F$ is the total angularmomentum quantum number and $m_{F}$ is its projection on the quantization axis. For the two-photon $1 \mathrm{~S}-2 \mathrm{~S}$ transition, the selection rules are $\Delta F=0$ and $\Delta m_{F}=0$ [28], which allows four 1S-2S transitions in hydrogen and four in antihydrogen. These transitions involve states with identical spin configurations. However, for hydrogen the result (10) of the perturbative calculation implies that the leading-order level shifts for $1 \mathrm{~S}$ and $2 \mathrm{~S}$ hydrogen states with the same spin configuration are identical. The same follows from Eq. (11) for antihydrogen. Therefore, the 1S-2S frequencies are unaffected at leading order for all these transitions. Indeed, this result could have been anticipated from the discussion in Ref. [9] showing that observable CPT-violating effects must also involve spin-flip processes and CT violation.

In summary, no leading-order $1 S$-2S spectroscopic signal occurs for Lorentz or CPT violation in free hydrogen or in free antihydrogen [11].

Non-leading level shifts can produce observable signals, but these are suppressed. The dominant subleading effects from electron/positron and proton/antiproton CPT- and Lorentz-violation terms are relativistic corrections suppressed by at least $\alpha^{2} \simeq 5 \times 10^{-5}$. As an explicit example, consider the coupling coefficient $b_{\mu}^{e}$ in Eq. (8). If this coupling is nonzero, the $m_{F}=0 \rightarrow m_{F^{\prime}}=0$ is unaffected but a subleading-order frequency shift in the $m_{F}=1 \rightarrow m_{F^{\prime}}=1$ transition appears. It is given by

$$
\delta \nu_{1 S-2 S}^{H} \approx-\frac{\alpha^{2} b_{3}^{e}}{8 \pi}
$$

The potential signals from subleading effects are suppressed to the extent that feasible $g-2$ experiments could exclude their observation in free hydrogen or antihydrogen. As mentioned above, an electron-positron $g-2$ comparison using present technology with a minor change in experimental procedure could attain a tight bound on $b_{3}^{e}$ [9]. The effect of a nonzero $b_{3}^{e}$ at this level on the $1 \mathrm{~S}-2 \mathrm{~S}$ frequency in free hydrogen would be to produce a nonzero frequency shift $\delta \nu_{1 S-2 S}^{H} \lesssim 5 \mu \mathrm{Hz}$, which is below the resolution of the 1S-2S line center. Similarly, bounds attainable in Penning-trap experiments comparing $g-2$ for protons and antiprotons could exclude observable signals in 1S-2S transitions. The basic reason why $g-2$ experiments are so effective in constraining possible violations is that they involve spin-flip transitions that exhibit unsuppressed sensitivity to CPT and Lorentz breaking. The $g-2$ experiments have an absolute frequency resolution of about $1 \mathrm{~Hz}$. Although the idealized line-center resolution for free-hydrogen or free-antihydrogen 1S-2S transitions is about three orders of magnitude better, the CPT- and Lorentz-violating effects on these transitions are suppressed by about five orders of magnitude and so the net sensitivity of the $g-2$ experiments is better. Note that it is inappropriate in this context to compare the conventional figure of merit for CPT breaking in $g-2$ experiments [29],

$$
r_{g}=\frac{\left|g_{e^{-}}-g_{e^{+}}\right|}{g_{\mathrm{av}}} \lesssim 2 \times 10^{-12}
$$

with the idealized resolution of the $1 \mathrm{~S}-2 \mathrm{~S}$ line,

$$
\Delta \nu_{1 S-2 S} / \nu_{1 S-2 S} \simeq 10^{-18}
$$

These two quantities are physically very different [9]. A relevant comparison would involve the same physics, such as the absolute frequency resolution and sensitivity to CPT- and Lorentz-violating effects used above. 


\section{S-2S SPECTROSCOPY IN TRAPPED HYDROGEN AND ANTIHYDROGEN}

The results in the previous section for free hydrogen and antihydrogen may be modified in the presence of external fields, which can induce transitions between states with different spin configurations. External fields are present for the class of proposed experiments [23] involving spectroscopy of hydrogen or antihydrogen confined within a magnetic trap with an axial bias magnetic field, such as an Ioffe-Pritchard trap [30]. Next, a theoretical analysis of possible signals of $\mathrm{CPT}$ and Lorentz violation in this context is described. In what follows, the four 1S hyperfine Zeeman levels in hydrogen are denoted by $|a\rangle_{1},|b\rangle_{1},|c\rangle_{1},|d\rangle_{1}$, in order of increasing energy in a magnetic field $B$. The corresponding four $2 \mathrm{~S}$ levels are denoted $|a\rangle_{2},|b\rangle_{2},|c\rangle_{2},|d\rangle_{2}$. The same notation is used for the $1 \mathrm{~S}$ and $2 \mathrm{~S}$ hyperfine Zeeman levels in antihydrogen.

The Zeeman levels $|a\rangle_{n}$ and $|c\rangle_{n}, n=1,2$, are mixed-spin states. For hydrogen, they are given in terms of the basis states $\left|m_{J}, m_{I}\right\rangle$ by

$$
\begin{aligned}
& |a\rangle_{n}=\cos \theta_{n}\left|-\frac{1}{2}, \frac{1}{2}\right\rangle-\sin \theta_{n}\left|\frac{1}{2},-\frac{1}{2}\right\rangle \quad, \\
& |c\rangle_{n}=\sin \theta_{n}\left|-\frac{1}{2}, \frac{1}{2}\right\rangle+\cos \theta_{n}\left|\frac{1}{2},-\frac{1}{2}\right\rangle .
\end{aligned}
$$

The mixing angles $\theta_{n}$ are given by

$$
\tan 2 \theta_{n} \approx \frac{(51 \mathrm{mT})}{n^{3} B}
$$

Expressions similar to (15) hold for antihydrogen, but the spin labels are reversed.

For hydrogen, in the absence of perturbations and prior to excitation, the low-field-seeker states $|c\rangle_{1}$ and $|d\rangle_{1}$ are confined in the trap. Spin-exchange collisions cause the $|c\rangle_{1}$ occupation to decrease with time: $|c\rangle_{1}+|c\rangle_{1} \rightarrow|b\rangle_{1}+|d\rangle_{1}$. The primary states in the trap are therefore $|d\rangle_{1}$. Moreover, the transition $|d\rangle_{1} \rightarrow|d\rangle_{2}$ is field independent for small magnetic fields. It might therefore seem reasonable to perform an experiment comparing the frequency $\nu_{d}^{H}$ for the 1S-2S transition $|d\rangle_{1} \rightarrow|d\rangle_{2}$ in hydrogen with the frequency $\nu_{d}^{\bar{H}}$ for the corresponding transition in antihydrogen. However, the $|d\rangle_{n}$ states in hydrogen have no spin mixing, so the frequency is unaffected to leading order by CPTand Lorentz-breaking effects. A similar result is true for antihydrogen. This means that [11]

$$
\delta \nu_{d}^{H}=\delta \nu_{d}^{\bar{H}} \simeq 0
$$

to leading order.

Thus, no leading-order $1 S$-2S spectroscopic signal for Lorentz or CPT violation occurs for unmixed-spin states in hydrogen or antihydrogen confined in a magnetic trap with an axial bias field [11].

It therefore appears worthwhile theoretically to examine $1 \mathrm{~S}-2 \mathrm{~S}$ transitions involving mixed-spin states. Indeed, for the $|c\rangle_{1} \rightarrow|c\rangle_{2}$ transition in hydrogen the spin mixing induces an unsuppressed frequency shift

$$
\delta \nu_{c}^{H} \approx-\frac{\kappa}{2 \pi}\left(b_{3}^{e}-b_{3}^{p}-d_{30}^{e} m_{e}+d_{30}^{p} m_{p}-H_{12}^{e}+H_{12}^{p}\right),
$$

where

$$
\kappa \equiv \cos 2 \theta_{2}-\cos 2 \theta_{1}
$$

The corresponding transition in antihydrogen in the same magnetic field exhibits a frequency shift $\delta \nu_{c}^{\bar{H}}$ given by an expression of the form (18) except with opposite signs for $b_{3}^{e}$ and $b_{3}^{p}$.

The unsuppressed sensitivity to CPT and Lorentz breaking of the $|c\rangle_{1} \rightarrow|c\rangle_{2}$ transition represents a theoretical advantage of a factor of about $4 / \alpha^{2} \simeq 10^{5}$ over the suppressed effects from the $|d\rangle_{1} \rightarrow|d\rangle_{2}$ transition. Since the frequencies $\nu_{c}^{H}$ and $\nu_{c}^{\bar{H}}$ vary with the spatial components of the CPT-violating couplings $b_{\mu}^{e}$ and $b_{\mu}^{p}$ in the comoving Earth frame, they would exhibit diurnal variations. Moreover, frequency measurements in a given magnetic trapping field would also display a nonzero difference

$$
\Delta \nu_{1 S-2 S, c} \equiv \nu_{c}^{H}-\nu_{c}^{\bar{H}} \approx-\frac{\kappa}{\pi}\left(b_{3}^{e}-b_{3}^{p}\right) .
$$

This difference varies with the amount of spin mixing according to the parameter $\kappa$, so a maximal value of $\kappa$ is theoretically desirable. It is $\kappa \simeq 0.67$, and it is attained when $B_{0} \simeq 0.01 \mathrm{~T}$.

From the experimental perspective, the $1 \mathrm{~S}-2 \mathrm{~S}$ transition $|c\rangle_{1} \rightarrow|c\rangle_{2}$ is likely to be less advantageous because it is field dependent in both hydrogen and antihydrogen. An experiment would therefore have to address the issue of 
Zeeman broadening from the inhomogeneous trapping fields. For instance, at $B \simeq 10 \mathrm{mT}$ the $1 \mathrm{~S}-2 \mathrm{~S}$ linewidth for the $|c\rangle_{1} \rightarrow|c\rangle_{2}$ transition is broadened to over $1 \mathrm{MHz}$ for both hydrogen and antihydrogen even at a temperature of $100 \mu \mathrm{K}$. Although present methods might reduce the impact of this effect, it would seem necessary that other techniques be developed if resolutions of the order of the natural linewidth are to be reached.

To summarize, unsuppressed $1 S$-2S spectroscopic signals for Lorentz and CPT violation appear for transitions involving mixed-spin states in hydrogen or antihydrogen atoms confined in a magnetic trap with an axial bias field [11].

\section{HYPERFINE SPECTROSCOPY IN HYDROGEN AND ANTIHYDROGEN}

The remainder of this talk addresses the issue of possible CPT- and Lorentz-violating signals in frequency measurements of hyperfine Zeeman transitions in trapped hydrogen and antihydrogen [11]. The interest in these is partially motivated by the resolution below $1 \mathrm{mHz}$ that has already been attained in transitions between $F=0$ and $F^{\prime}=1$ hyperfine levels of a hydrogen maser [31].

Perturbative calculations along the lines described above show that all four hyperfine levels in the 1S ground state of hydrogen are shifted by CPT- and Lorentz-violating effects. One contribution to the shifts, $a_{0}^{e}+a_{0}^{p}-c_{00}^{e} m_{e}-c_{00}^{p} m_{p}$, is identical for all four levels and therefore has no effect on any frequencies. There are also spin-dependent energy shifts, given by [11]

$$
\begin{aligned}
& \Delta E_{a}^{H} \approx \hat{\kappa}\left(b_{3}^{e}-b_{3}^{p}-d_{30}^{e} m_{e}+d_{30}^{p} m_{p}-H_{12}^{e}+H_{12}^{p}\right) \\
& \Delta E_{b}^{H} \approx b_{3}^{e}+b_{3}^{p}-d_{30}^{e} m_{e}-d_{30}^{p} m_{p}-H_{12}^{e}-H_{12}^{p} \\
& \Delta E_{c}^{H} \approx-\Delta E_{a}^{H} \\
& \Delta E_{d}^{H} \approx-\Delta E_{b}^{H}
\end{aligned}
$$

where

$$
\hat{\kappa} \equiv \cos 2 \theta_{1}
$$

is a parameter analogous to $\kappa$ of Eq. (19) that grows with $B$, with $\hat{\kappa} \simeq 1$ when $B \simeq 0.3 \mathrm{~T}$.

To begin, suppose the magnetic field vanishes. Then, $\hat{\kappa}=0$ and so Eq. (21) shows that the states $|a\rangle_{1}$ and $|c\rangle_{1}$ are unchanged. However, the energies of $|b\rangle_{1}$ and $|d\rangle_{1}$ shift equally in magnitude but oppositely in sign. Thus, even for $B=0$ the three $F=1$ levels are split.

If instead the magnetic field is nonzero, then the energies of all four hyperfine levels are changed. Consider first the conventional hydrogen maser, which uses a small magnetic field and involves the (approximately field-independent) transition $|c\rangle_{1} \rightarrow|a\rangle_{1}$. For this situation, the value of $\hat{\kappa}$ is roughly $10^{-4}$ so the spin-mixing is small. This would act as a suppression factor for CPT- and Lorentz-breaking effects in possible high-precision measurements of the maser $\sigma$ line $|c\rangle_{1} \rightarrow|a\rangle_{1}$.

In contrast, unsuppressed frequency differences appear between the field-dependent transitions $|d\rangle_{1} \rightarrow|a\rangle_{1}$ and $|b\rangle_{1} \rightarrow|a\rangle_{1}$. Equation (21) gives

$$
\left|\Delta \nu_{d-b}^{H}\right| \approx \frac{1}{\pi}\left|b_{3}^{e}+b_{3}^{p}-d_{30}^{e} m_{e}-d_{30}^{p} m_{p}-H_{12}^{e}-H_{12}^{p}\right| .
$$

This difference would vary diurnally in the comoving Earth frame, as occurs with the shifts (18), so in principle a measurement of $\left|\Delta \nu_{d-b}^{H}\right|$ in hydrogen alone could provide a signal of CPT and Lorentz violation. However, in practice the attainable frequency resolution is likely to be affected by the broadening due to field inhomogeneities. An experiment of this type would also need to address the issue of distinguishing the signal from possible backgrounds due to residual Zeeman splittings.

Instead, one could envisage using a field-independent transition point to minimize the frequency dependence on the magnetic field and making a direct comparison of hydrogen and antihydrogen transition frequencies to avoid issues with the background splittings. Consider, for example, an experiment performing high-resolution radiofrequency spectroscopy in trapped hydrogen and antihydrogen on the $|d\rangle_{1} \rightarrow|c\rangle_{1}$ transition at the field-independent transition point $B \simeq 0.65 \mathrm{~T}$. To avoid Doppler broadening, cooling to temperatures of $100 \mu \mathrm{K}$ with a good signal-to-noise ratio is likely to be needed. Also, the relatively high bias field suggests potentially larger field inhomogeneities would occur, so a stiff box shape would be preferable for the trapping potential. Under these circumstances, it may be possible to attain frequency resolutions of order $1 \mathrm{mHz}$.

In a magnetic field of $0.65 \mathrm{~T}$, the state $|c\rangle_{1}$ in hydrogen is well approximated as a spin-polarized level with $\left|m_{J}, m_{I}\right\rangle=|1 / 2,-1 / 2\rangle$. This means that the transition of interest, $|d\rangle_{1} \rightarrow|c\rangle_{1}$, involves a proton spin flip, which 
in turn implies a signal dependence only on CPT- and Lorentz-violating effects for the proton. Explicit calculation shows that the frequency shift for hydrogen is

$$
\delta \nu_{c \rightarrow d}^{H} \approx \frac{1}{\pi}\left(-b_{3}^{p}+d_{30}^{p} m_{p}+H_{12}^{p}\right)
$$

while that for antihydrogen is

$$
\delta \nu_{c \rightarrow d}^{\bar{H}} \approx \frac{1}{\pi}\left(b_{3}^{p}+d_{30}^{p} m_{p}+H_{12}^{p}\right)
$$

confirming the expected dependence on proton coupling coefficients.

Like the quantity $\left|\Delta \nu_{d-b}^{H}\right|$ of Eq. (23), diurnal variations of the the frequencies $\nu_{c \rightarrow d}^{H}$ and $\nu_{c \rightarrow d}^{\bar{H}}$ would provide a signal for CPT and Lorentz violation. However, the difference

$$
\Delta \nu_{c \rightarrow d} \equiv \nu_{c \rightarrow d}^{H}-\nu_{c \rightarrow d}^{\bar{H}} \approx-\frac{2 b_{3}^{p}}{\pi}
$$

between these frequencies has the potential to provide an instantaneous, clean, and accurate test of CPT-violating couplings $b_{3}^{p}$ for the proton.

Relevant figures of merit for the diurnal and instantaneous signals in Eqs. (24), (25), and (26) (as well as ones for other signals mentioned above in this and earlier sections) can be defined following the methods developed for Penning-trap tests [9]. For the instantaneous signal in Eq. (26), an appropriate choice is

$$
\begin{aligned}
r_{r f, c \rightarrow d}^{H} & \equiv \frac{\left|\left(\mathcal{E}_{1, d}^{H}-\mathcal{E}_{1, c}^{H}\right)-\left(\mathcal{E}_{1, d}^{\bar{H}}-\mathcal{E}_{1, c}^{\bar{H}}\right)\right|}{\mathcal{E}_{1, \mathrm{av}}^{H}} \\
& \approx \frac{2 \pi\left|\Delta \nu_{c \rightarrow d}\right|}{m_{H}},
\end{aligned}
$$

where $m_{H}$ is the atomic mass of hydrogen and where the relativistic energies in the ground-state hyperfine levels are denoted by $\mathcal{E}_{1, d}^{H}, \mathcal{E}_{1, c}^{H}$ for hydrogen and by $\mathcal{E}_{1, d}^{\bar{H}}, \mathcal{E}_{1, c}^{\bar{H}}$ for antihydrogen. Suppose, for instance that a $1 \mathrm{mHz}$ frequency resolution could indeed be reached in an experiment of this type. This would represent an estimated upper bound on the figure of merit (27) of approximately $r_{r f, c \rightarrow d}^{H} \lesssim 5 \times 10^{-27}$. The associated constraint on the coefficient $b_{3}^{p}$ would be $\left|b_{3}^{p}\right| \lesssim 10^{-18} \mathrm{eV}$. This is more than four orders of magnitude better than bounds attainable from 1S-2S transitions and roughly three orders of magnitude better than estimated attainable bounds [9] from $g-2$ experiments in Penning traps.

To summarize, unsuppressed Zeeman hyperfine spectroscopic signals for Lorentz or CPT violation appear for transitions involving spin-flip hyperfine states in hydrogen and antihydrogen atoms confined in a magnetic trap with an axial bias field [11].

\section{ACKNOWLEDGMENTS}

V.A.K. thanks Orfeu Bertolami, Don Colladay, Rob Potting, Stuart Samuel, and Rick Van Kooten for collaborations leading to some of the results described in this talk. This work is supported in part by the Department of Energy under grant number DE-FG02-91ER40661 and by the National Science Foundation under grant number PHY-9503756.

\section{REFERENCES}

1. V.A. Kostelecký and S. Samuel, Phys. Rev. Lett. 63 (1989) 224; ibid., 66 (1991) 1811; Phys. Rev. D 39 (1989) $683 ;$ ibid., 40 (1989) 1886; V.A. Kostelecký and R. Potting, Nucl. Phys. B 359 (1991) 545; Phys. Lett. B 381 (1996) 89.

2. The discrete symmetries C, P, T are discussed, for example, in R.G. Sachs, The Physics of Time Reversal (University of Chicago Press, Chicago, 1987).

3. D. Colladay and V.A. Kostelecký, Phys. Rev. D 55 (1997) 6760; preprint IUHET 359, Phys. Rev. D in press (hep$\mathrm{ph} / 9809521)$.

4. B. Schwingenheuer et al., Phys. Rev. Lett. 74 (1995) 4376; L.K. Gibbons et al., Phys. Rev. D 55 (1997) 6625 ; R. Carosi et al., Phys. Lett. B 237 (1990) 303. 
5. V.A. Kostelecký and R. Potting, in D.B. Cline, ed., Gamma Ray-Neutrino Cosmology and Planck Scale Physics (World Scientific, Singapore, 1993) (hep-th/9211116); Phys. Rev. D 51 (1995) 3923; D. Colladay and V. A. Kostelecký, Phys. Lett. B 344 (1995) 259; Phys. Rev. D 52 (1995) 6224; V.A. Kostelecký and R. Van Kooten, Phys. Rev. D 54 (1996) 5585 .

6. OPAL Collaboration, R. Ackerstaff et al., Z. Phys. C 76 (1997) 401; DELPHI Collaboration, M. Feindt et al., preprint DELPHI 97-98 CONF 80 (July 1997).

7. V.A. Kostelecký, Phys. Rev. Lett. 80 (1998) 1818.

8. P.B. Schwinberg, R.S. Van Dyck, Jr., and H.G. Dehmelt, Phys. Lett. A 81 (1981) 119; Phys. Rev. D 34 (1986) 722 ; L.S. Brown and G. Gabrielse, Rev. Mod. Phys. 58 (1986) 233; R.S. Van Dyck, Jr., P.B. Schwinberg, and H.G. Dehmelt, Phys. Rev. Lett. 59 (1987) 26; G. Gabrielse et al., ibid., 74 (1995) 3544.

9. R. Bluhm, V.A. Kostelecký and N. Russell, Phys. Rev. Lett. 79 (1997) 1432; Phys. Rev. D 57 (1998) 3932.

10. See, for example, M. Charlton et al., Phys. Rep. 241 (1994) 65; J. Eades, ed., Antihydrogen (J.C. Baltzer, Geneva, 1993).

11. R. Bluhm, V.A. Kostelecký and N. Russell, Indiana University preprint IUHET 388 (1998).

12. O. Bertolami et al., Phys. Lett. B 395 (1997) 178.

13. V.A. Kostelecký and C.D. Lane, in preparation.

14. V.W. Hughes, H.G. Robinson, and V. Beltran-Lopez, Phys. Rev. Lett. 4 (1960) 342; R.W.P. Drever, Philos. Mag. 6 (1961) 683; J.D. Prestage et al., Phys. Rev. Lett. 54 (1985) 2387; S.K. Lamoreaux et al., ibid., 57 (1986) 3125; T.E. Chupp et al., ibid., 63 (1989) 1541.

15. S.M. Carroll, G.B. Field, and R. Jackiw, Phys. Rev. D 41 (1990) 1231.

16. P. Haves and R.G. Conway, Mon. Not. R. Astr. Soc. 173 (1975) 53P; J.N. Clarke, P.P. Kronberg and M. SimardNormandin, ibid., 190 (1980) 205.

17. B. Nodland and J.P. Ralston, Phys. Rev. Lett. 78 (1997) 3043; Phys. Rev. Lett. 79 (1997) 1958 ; astro-ph/9706126.

18. S.M. Carroll and G.B. Field, Phys. Rev. Lett. 79 (1997) 2394; D.J. Eisenstein and E.F. Bunn, Phys. Rev. Lett. 79 (1997) 1957; J.P. Leahy, astro-ph/9704285; J.F.C. Wardle, R.A. Perley and M.H. Cohen, Phys. Rev. Lett. 79 (1997) $1801 ;$ T.J. Loredo, E.E. Flanagan and I.M. Wasserman, Phys. Rev. D 56 (1997) 7507.

19. S. Coleman and S. Glashow, Phys. Lett. B 405 (1997) 249.

20. G. Baur et al., Phys. Lett. B 368 (1996) 251.

21. G. Blanford et al., Phys. Rev. Lett. 80 (1998) 3037.

22. G. Blanford et al., Phys. Rev. D 57 (1998) 6649.

23. B. Brown et al., Nucl. Phys. B (Proc. Suppl.) 56A (1997) 326; M.H. Holzscheiter et al., ibid., 336.

24. T. Udem et al., Phys. Rev. Lett. 79 (1997) 2646.

25. C.L. Cesar et al., Phys. Rev. Lett. 77 (1996) 255.

26. See, for example, T.W. Hänsch, in D.J. Wineland, C.E. Wieman, and S.J. Smith, eds., Atomic Physics 14 (A.I.P. New York, 1995).

27. See, for example, G. Breit, Phys. Rev. 34 (1929) 553.

28. B. Cagnac, G. Grynberg, and F. Biraben, J. Physique 34 (1973) 845.

29. See, for example, R.M. Barnett et al., Review of Particle Properties, Phys. Rev. D 54 (1996) 1.

30. Y.V. Gott, M.S. Ioffe, and V.G. Tel'kovskii, Nucl. Fusion, Suppl. Pt. 3 (1962) 1045; D.E. Pritchard, Phys. Rev. Lett. 51 (1983) 1336.

31. N.F. Ramsey, Physica Scripta T59 (1995) 323. 